\title{
Charge order and phase segregation in overdoped bilayer manganites
}

\author{
T. Maitra ${ }^{1 *}$ A. Taraphder ${ }^{2}$ and H.Beck ${ }^{3 \dagger}$ \\ ${ }^{1}$ Institut für Theoretische Physik, J. W. Goethe Universität, \\ Max-von-Laue-Strasse 1, 60438 Frankfurt am Main, Germany \\ ${ }^{2}$ Department of Physics 83 Meteorology and Centre for Theoretical Studies, \\ Indian Institute of Technology, Kharagpur 721302 India \\ ${ }^{3}$ Institute of Physics, University of Neuchatel, rue de Breguet 1, CH-2000 Switzerland
}

\begin{abstract}
There have been recent reports of charge ordering around $x=0.5$ in the bilayer manganites. At $x=0.5$, there appears to be a coexistence region of layered A-type antiferromagnetc and charge order. There are also reports of orbital order in this region without any Jahn-Teller effect. Based on physical grounds, this region is investigated from a model that incorporates the two $e_{g}$ orbitals at each Mn site and a near-neighbour Coulomb repulsion. It is shown that there indeed is both charge and orbital order close to the half-doped region coincident with a layered magnetic structure. Although the orbital order is known to drive the magnetic order, the layered magnetic structure is also favoured in this system by the lack of coherent transport across the planes and the reduced dimensionality of the lattice. The anisotropic hopping across the $e_{g}$ orbitals and the underlying layered structure largely determine the orbital arrangements in this region, while the charge order is primarily due to the long range interactions.
\end{abstract}

PACS numbers: 75.47.Gk, 75.30.Et

\section{INTRODUCTION}

Bilayer manganites such as $\mathrm{La}_{2-2 x} \mathrm{Sr}_{1+2 x} \mathrm{Mn}_{2} \mathrm{O}_{7}$, the $n=2$ member of the Ruddelsden-Popper series $(R, A)_{n+1} M n_{n} O_{3 n+1}$ (where $R$ and $A$ are rareearth and alkaline-earth ions respectively), show ${ }^{1}$ a distinct lack of symmetry in the nature of their ground states across $x=0.5$ just as their $3 \mathrm{D}$ counterparts. These systems have started drawing attention after colossal magnetoresistance (CMR) has been reported in them ${ }^{2}$. These layered systems not only show large magnetoresistance and a sequence of magnetic phases 3.4 , they are very rich in their charge, magnetic and orbital structures. Most of the studies in the bilayer system so far focussed on the $\mathrm{Mn}^{3+}$ richer region of $x<0.5$. The $\mathrm{Mn}^{4+}$-rich region $(x>0.5)$ is now explored carefully both experimentally ${ }^{1.5 .6}$ and theoretically ${ }^{7.8}$ and a succession of magnetic phases $\mathrm{A} \rightarrow \mathrm{C} \rightarrow \mathrm{G}$ has been confirmed. The situation very close to and around $x=0.5$ is somewhat poorly understood yet. It emerged recently that there is a coexistence between charge ordered and layered A-type spin ordered state 5.9 there, giving way to the $\mathrm{C}$-type (or its polytype $\mathrm{e}^{\frac{1}{}}$ ) magnetic order at larger $\mathrm{Mn}^{4+}$ concentration. Both A-type (ferromagnetic layers coupled antiferromagnetically)

\footnotetext{
*email: maitra@itp.uni-frankfurt.de

$\dagger$ email: Hans.Beck@unine.ch
}

and C-type (ferromagnetic chains coupled antiferromagnetically) phases have been found to be orbitally ordered.

There are strong indications from several experimental groups that at and around $x=0.5$, a charge ordered state coexists with an A-type antiferromagnetic phase. Both neutron scattering ${ }^{10.11}$ and muon spin rotation corroborate this view. A discontinuity in the muon precession frequency at $x=0.52$ has been observed by Coldea et al. $\stackrel{5}{.}$. Recently Wilkins et al. 12 have reported that although there is clear evidence of both charge and orbital order in $\mathrm{La}_{2-2 x} \mathrm{Sr}_{1+2 x} \mathrm{Mn}_{2} \mathrm{O}_{7}$ at $x=0.5$, there is no detectable signature for any Jahn-Teller (JT) distortion in their resonant X-ray diffraction study. JT distortion of the $\mathrm{MnO}_{6}$ octahedra begin to appear beyond $x=0.55 \underline{\underline{6}}$. There is, though, a reasonably large (tetragonal) static distortion - the crystallographic $c / a$ ratio reaches a minimum 1 around the same composition, enhancing the AF super-exchange along $c$ direction. This is expected to favour the A-type spin order in that region. Unlike in the $3 \mathrm{D}$ manganites, there is no significant buckling of the bonds during distortion.

It is now generally believed that the various magnetic structures owe their origin to a large degree to the underlying orbital order ${ }^{7.13}$. Models have been proposed ${ }^{14.15}$ for the manganites that incorporate the $e_{g}$ orbitals and the anisotropic hopping between them. The use of such models to the bilayer manganites (like $\mathrm{La}_{2-2 x} \mathrm{Sr}_{1+2 x} \mathrm{Mn}_{2} \mathrm{O}_{7}$ ) has only had limited success ${ }^{8}$ though, the A-phase is overestimated owing to the low 
dimensionality of the lattice. A much improved magnetic and orbital phase diagram was later obtained ${ }^{7}$ from a model that included the JT distortion as well as the proper choice of orbital degrees of freedom. The A-type AFM instability is indeed quite strong in the layered system ${ }^{1}$, extending from $x=0.42$ to 0.66 and at low temperatures. The FM region of the phase diagram of some of the 3D manganites around $x=0.5$ is absent here, taken over by the A-type AFM phase. Having only two layers separated by large distance from the adjoining bilayer in the c-direction impedes a long range charge transport in that direction leading to stronger $\mathrm{AF}$ correlations along c-direction thereby contributing to an enhanced A-type instability. Beyond $x=0.66$, it is replaced by the C-type (and its polytype) order. The A- and C-phases are orbitally ordered and there is a connection between the preferred orbital order and the observed magnetic order.

However, the presence of charge order and its relation to the underlying magnetic phases have not been investigated in any of the previous theoretical studies.8. in bilayer systems. The question of charge order and possible phase separation in the overdoped bilayer systems, therefore, still remains an open issue with increasing evidence in favour of such coexistence coming from the experiments. We attempt to address it in the following, starting from a model that incorporates the essential physical attributes of this region.

\section{THE MODEL}

A quite general model for the bilayer manganites has been used 7 to delineate the different orbital and magnetic structures for $x>0.5$. It incorporates the degenerate $e_{g}$ manifold and the physics of double exchange (DE) along with electron-electron interactions. This model can be adapted to investigate the charge order by including a longer range Coulomb term in the interaction part. Such a term has been known ${ }^{20.21}$ to give rise to coexisting charge ordered state in the $3 \mathrm{D}$ manganites at $x=0.5$.

$$
H=J_{A F} \sum_{<i j>} \mathbf{S}_{\mathbf{i}} \cdot \mathbf{S}_{\mathbf{j}}-J_{H} \sum_{i} \mathbf{S}_{\mathbf{i}} \cdot \mathbf{S}_{\mathbf{i}}-\sum_{<i j>\sigma, \alpha, \beta} t_{i, j}^{\alpha \beta} c_{i, \alpha, \sigma}^{\dagger} c_{j, \beta, \sigma}+H_{i n t}
$$

As usual the charge and spin dynamics of the conventional $\mathrm{DE}$ mode ${ }^{18}$ operate here too, with additional degrees of freedom coming from the degenerate $e_{g}$ orbitals ( $\alpha, \beta$ stand for the $e_{g}$ orbitals) and hopping across them, which is determined by their symmetry. In the reasonably large Hund's coupling limit, which is relevant for the studies on manganites, the DE mechanism implies that an electron can hop onto a site if core spins at that site (as well as the spin of any $e_{g}$ electron there) are parallel to its spin. Mobility reduces drastically with increasing $\mathrm{J}_{H}$ if they are antiparallel. In Eqn.(1) $\mathbf{S}_{\mathbf{i}}$ and $\mathbf{s}_{\mathbf{i}}$ are the $t_{2 g}$ and $e_{g}$ spins at site $i$ and $J_{H}$ and $J_{A F}$ are the Hund and super-exchange (SE) coupling respectively. $H_{i n t}=U^{\prime} \sum_{i \sigma \sigma^{\prime}} \hat{n}_{i 1 \sigma} \hat{n}_{i 2 \sigma^{\prime}}+V \sum_{i} \hat{n}_{i} \hat{n}_{j}$ contains the on-site inter-orbital and the near-neighbour Coulomb repulsion term. The intra-orbital term can be ignored as $J_{H}$ in manganites is fairly large, preventing double occupancy in any given Mn $3 \mathrm{~d}$ orbital ${ }^{16}$. The exchange interaction between two bilayers is known to be at least a 100 times weaker ${ }^{17}$ than the intra-bilayer exchange. Two bilayers are also well-separated in an unit cell and intervened by the rare-earth ions. One can, therefore, use only one bilayer for a reasonable description of the system ${ }^{8}$. In general, in manganites, there is a strong JT coupling and that is included in the Hamiltonian. As we mentioned above, in the bilayer systems (e.g. in $\mathrm{La}_{2-2 x} \mathrm{Sr}_{1+2 x} \mathrm{Mn}_{2} \mathrm{O}_{7}$ ), the JT coupling is weak and does not play a major role $\underline{6.12}$ in the region $x<0.6$. In the absence of electronlattice coupling, the kinetic energy (KE) of electrons in the $e_{g}$ band and the Hund's coupling between $t_{2 g}$ and $e_{g}$ spins compete with the antiferromagnetic $\mathrm{SE}$ interaction leading to a variety of magnetic and orbital structures. Considering that there are two $e_{g}$ orbitals, the nominal band filling is $\frac{1-x}{4}$.

Typical values of the interaction and band parameters for the bilayer systems are in the same range as in the 3D manganites. As in their 3D counterparts, the Hund coupling and Coulomb correlations are the largest scale of energy 16 in the problem. We neglect the inter-orbital Coulomb term in the following discussion of charge order. The inter-orbital Coulomb term does not have strong effect in the doping range 
$x$ less than or around 0.5 primarily because of the low filling $((1-x) / 4)$ making two orbitals at the same site less likely to be occupied simultaneously. As we see below, the magnetic order also prefers preferential occupancies in one of the two $e_{g}$ orbitals in both A- and C-phases and the $U^{\prime}$ term only enhances that (discussed later).

Treating the $\mathrm{t}_{2 g}$ spins semiclassically ${ }^{18}$, the SE contribution to the ground state energy becomes $E_{S E}=$ $\frac{J_{A F} S_{0}^{2}}{2}\left(2 \cos \theta_{x y}+\cos \theta_{z}\right)$ where $\theta_{x y}$ and $\theta_{z}$ are the angle between the near-neighbour $t_{2 g}$ spins in the $x y$ plane and $z$ direction respectively. In the Ferromagnetic state, $\theta_{x y}=\theta_{z}=0$ while in the A-phase $\theta_{x y}=0$ and $\theta_{z}=\pi$.

In the limit of infinite $\mathrm{J}_{H}$, the $e_{g}$ electron quantization axes at each site $i$ is rotated in the local coordinate frame to make it parallel to $\mathbf{S}_{i}$. This is accomplished quite easily by the spin- $1 / 2$ rotation matrix $\exp \left(i \frac{\phi_{i}}{2} \sigma_{z}\right) \exp \left(i \frac{\theta_{i}}{2} \sigma_{y}\right) \exp \left(-i \frac{\phi_{i}}{2} \sigma_{z}\right)$ operating on a two component spinor. Allowing the core spins at each site to cant in the $x z$-plane and neglecting the phase term (the Berry phase) appearing from the transformation, it is straightfoward to show $\frac{18}{18}$ that the effective hopping matrix elements are $t_{x y}=t \cos \left(\theta_{x y} / 2\right)$ and $t_{z}=t \cos \left(\theta_{z} / 2\right)$. In this level of approximation, the diagonalisation of the KE part of $H$ reduces to solving the $2 \times 2$ matrix equation $\left\|\epsilon_{\alpha \beta}-\omega \delta_{\alpha \beta}\right\|=0$ for a system of spinless fermions. The matrix elements are obtained from the standard table of the overlap integrals $\frac{19}{19}$ involving $d_{x^{2}-y^{2}}$ and $d_{3 z^{2}-r^{2}}$ orbitals on neighbouring sites of a square lattice and the dispersions are

$$
\begin{gathered}
\epsilon_{11}=-2 t_{x y}\left(\cos k_{x}+\cos k_{y}\right) \\
\epsilon_{12}=t_{21}=-\frac{2}{\sqrt{3}} 2 t_{x y}\left(\cos k_{x}-\cos k_{y}\right) \\
\epsilon_{22}=-\frac{2}{3} t_{x y}\left(\cos k_{x}+\cos k_{y}\right)-\frac{8}{3} t_{z} \cos k_{z} .
\end{gathered}
$$

Using the form of $t_{x y}$ and $t_{z}$ in terms of $\theta_{x y}$ and $\theta_{z}$ in the $\mathrm{J}_{H} \rightarrow \infty$ approximation, the two energy bands are obtained by the diagonalization of the above matrix. In the uncanted $\mathrm{A}$ - and $\mathrm{C}$-phases, the dispersions for A- and C-phases become two- and one-dimensional respectively. In the pure FM phase, the DOS is three dimensional. However, in the bilayer system, owing to the absence of dispersion in the $z$ direction, the DOS in both FM and A phases show two dimensional character as shown in Fig. 1. There being only two

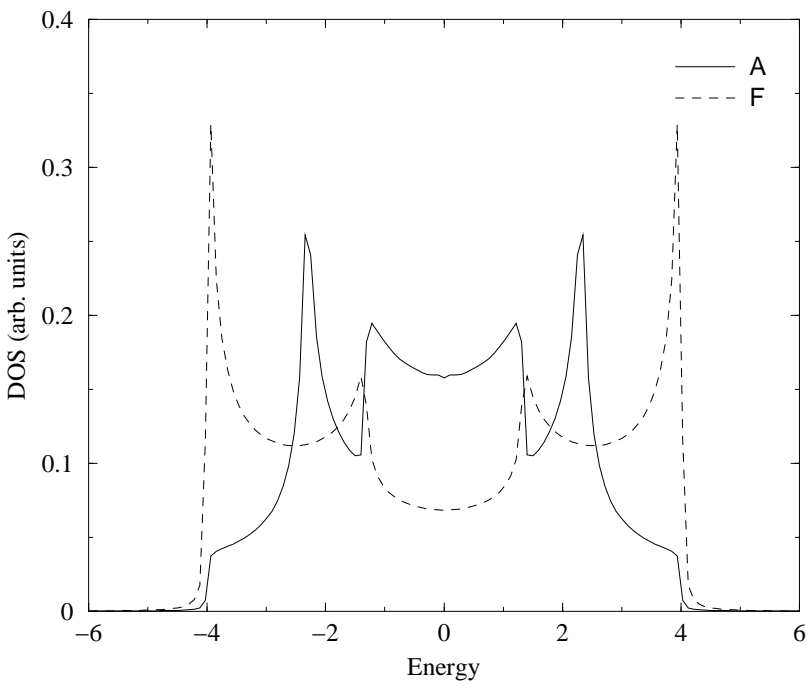

FIG. 1: Density of states in pure FM and A phase.

$k$-points in the $z$-direction, the DOS for the C-phase has two delta functions centred at $\pm \frac{8 t_{z}}{3}$. In all the calculations that follow, all energies are measured in terms of the overlap between the $d_{3 z^{2}-r^{2}}$ orbitals along $z$-direction $t^{\hat{z}}=t$, which has a typical value about $0.25 \mathrm{eV}$ in manganites.

\section{CHARGE AND ORBITAL ORDERING}

In order to look for charge ordering in the bilayer manganites, we treat the nearest neighbour Coulomb interaction $V \sum_{<i j>} \hat{n}_{i} \hat{n}_{j}$ in the mean field approximation, $\left\langle\hat{n_{i}}\right\rangle=n+C_{0} \exp \left(i Q . r_{i}\right)$ where $C_{0}$ is the charge order parameter and $n$ is the average number of electrons per site. We take the usual staggered ordering $Q=(\pi, \pi, \pi)$. The charge order parameter $C_{0}$ is then calculated self-consistently. The mean-field approximation is known to work quite well ${ }^{8.15 .16}$ for the ground state properties in the manganites. In the infinite $J_{H}$ limit, the electronic part of the Hamiltonian is a $4 \times 4$ matrix $\sum_{\mathbf{k}, \alpha \beta} \epsilon_{\mathbf{k} \alpha \beta} \tilde{c}_{\mathbf{k} \alpha}^{\dagger} \tilde{c}_{\mathbf{k} \beta}-\Delta \sum_{\mathbf{k}, \alpha} \tilde{c}_{\mathbf{k} \alpha}^{\dagger} \tilde{c}_{\mathbf{k}+\mathbf{Q} \alpha}$ (where $\tilde{c}$ represents the locally rotated $e_{g}$ electron operators described earlier) and this is diagonalized at each of the $\mathbf{k}$ points on a momentum grid.

The bands obtained thereof are filled upto a chemical potential and the order parameter $\Delta=z V C_{0}(z$ is the number of nearest neighbour) calculated along with the filling. The process is repeated until selfconsistency is achieved as is customary in the meanfield theory. Charge order is indeed observed in a re- 


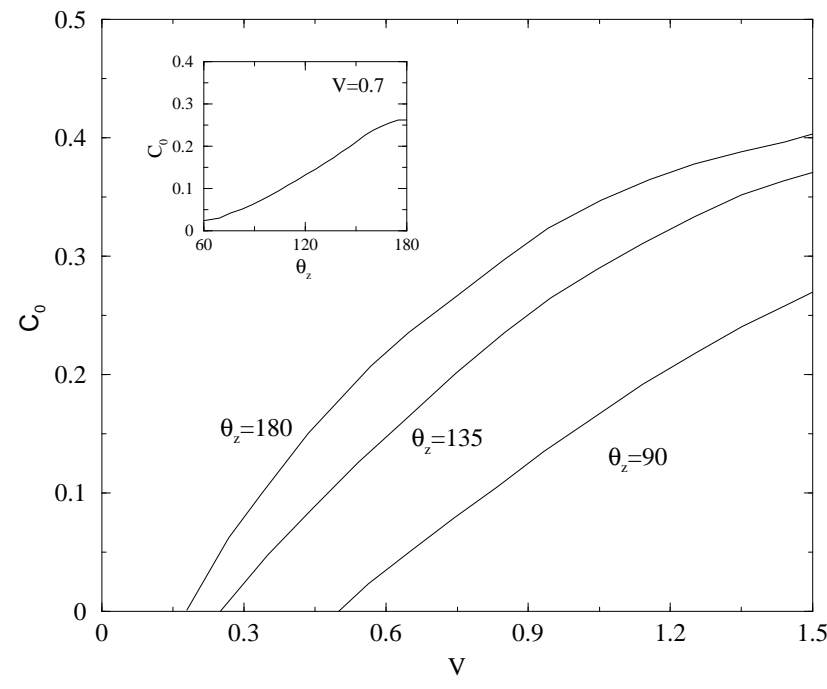

FIG. 2: Charge order parameter as a function of $V$ for three different angles $\theta_{z}$ in the limit $J_{H} \rightarrow \infty$. In the inset is shown the variation of charge order parameter with $\theta_{z}$ for a fixed $V=0.7$.

gion $x \geq 0.5$ when $V$ reaches a critical value, similar to the 3D manganites 20.21 . But unlike in the 3D case, we do not observe any F-phase coexisting with the $\mathrm{CO}$ in this region. As reported in previous work ${ }^{8,22}$ A-phase instability is quite strong in the layered manganites owing to the 2D structure of its DOS and even for $V=0$, there is no F-phase for $x \geq 0.5^{\stackrel{7}{?} \text {. The }}$ A-CO coexistence region extends in a region above $x=0.5$ (discussed later). In this limit of infinite $J_{H}$, the value of $V$ for which the CO phase appears depends strongly on the canting angle $\theta_{z}$ away from the A-phase (towards an F-phase). In Fig. 2 is shown the the dependence of the $\mathrm{CO}$ order parameter on $V$ at three different angles $\left(\theta_{z}=180 \mathrm{deg}\right.$ being the pure Aphase). The inset shows how $C_{0}$ varies with canting at a typical $V=0.7$. In the infinite Hund's coupling limit, the only way an electron gains $\mathrm{KE}$ in and $\mathrm{AF}$ configuration of background spins is via the canting of them ${ }^{23}$, thereby generating an effective non-zero hopping across. This has been observed ${ }^{15}$ in the $3 \mathrm{D}$ manganites as well close to its G-C phase boundary (for $x \simeq 1$ ). As we observe here, a CO phase does not coexist with an $\mathrm{F}$ phase (which is energetically unfavourable to an A-phase in this region) and therefore the increased canting requires larger $V$ to bring about the charge ordering. At a fixed $V$, therefore, the $\mathrm{CO}$ order parameter reduces with increased canting away from A-phase.
The appropriate limit for any of the manganites is, of course, a large but finite value of the Hund's coupling as is generally believed. In this limit, we can treat the core spins semi-classically again and the spin degrees of freedom for the $e_{g}$ electrons are reintroduced. In order to take care of this, the second term in the Hamiltonian (1) would have to be treated now in various possible ground states. We choose $\mathbf{S}_{i}=\mathbf{S}_{0} \exp \left(i \mathbf{q} \cdot \mathbf{r}_{\mathbf{i}}\right)$ to represent a homogeneous spin configuration, where $\mathbf{q}$ determines different spin arrangements for the $t_{2 g}$ spins ${ }^{20}$. The second term in eqn.(1) becomes $-J_{H} S_{0} \sum_{\mathbf{k} \alpha \sigma} \sigma c_{\mathbf{k} \alpha \sigma}^{\dagger} c_{\mathbf{k}+\mathbf{q} \alpha \sigma}$. The near-neighbour Coulomb term is treated in the meanfield as above. In this semi-classical approximation for the $t_{2 g}$ spins the Hamiltonian (1) reduces, then, to a $16 \times 16$ matrix ${ }^{20}$ at each $\mathbf{k}$ point.

This mean-field Hamiltonian is again diagonalised at each k-point on the momentum grid. The ground state energy is calculated for different magnetic structures. The $\mathrm{CO}$ order parameter is also determined self-consistently. Four different magnetic structures are relevant for the experimental phase diagram (with $\mathrm{q}$ values in the parentheses), A-type $(0,0, \pi)$, C-type $(\pi, \pi, 0)$ - the usual C-phase with FM chains along cdirection, and the 3D AFM G-type $(\pi, \pi, \pi)$. There is also the $\mathrm{C}^{\prime}$-type polytype structure ${ }^{1.7}$, with $\mathbf{q}=$ $(\pi, 0, \pi)$ This is same as a C-type, only that its FM ordering is along y-direction as reported by Ling et al. The magnetic structure with minimum ground state energy and the $\mathrm{CO}$ order parameter are determined for each set of parameters $\left(x, J_{H}, J_{A F}\right)$ for the range of doping $(0.5<x \leq 1)$.

Charge order is observed in the region $0.67>x \geq$ 0.5. Fig. 3 shows the $\mathrm{CO}$ order parameter as a function of $V$ for $x=0.5$. The underlying spin order in this region is found to be A-type and there is a A-CO to A transition at around $x=0.67$ for $V=0.7$. There is a jump in the $\mathrm{CO}$ order parameter as a function of $x$, shown in Fig. 4, indicating a first order transition. The coexistence region of $\mathrm{CO}$ and $\mathrm{A}$ is a manifestation of this first order transition. Based on the observed variation of these different charge and spin ordered states, we obtained a phase diagram in the $x-J_{H}$ plane for $V=0.7$.

The observed phase diagram, shown in Fig. 5, is markedly similar to the one obtained by Ling and coworkers. In addition it also has the charge ordered phase coexisting with the A-phase in the region close to $x=0.5$. As reported by Coldea et al. $\frac{5}{,}$, there is indeed a region of A-CO coexistence between $x=0.5$ and $x=0.65$. We note that we have also tried a charge order with wave vector $\mathbf{Q}=(\pi, \pi, 0)$ instead of the fully staggered one and observed that energetically 


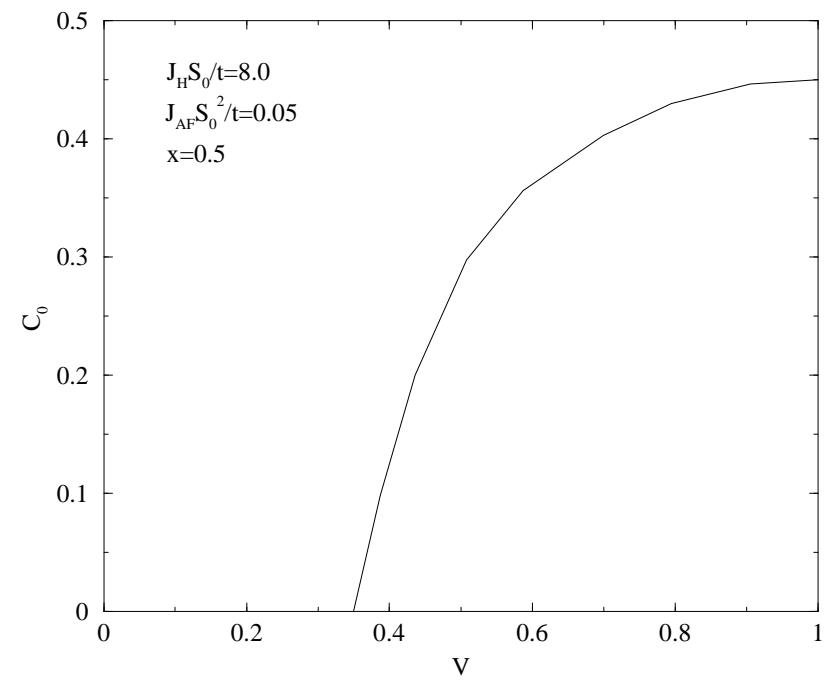

FIG. 3: Charge order parameter as a function of $V$ at $x=0.5$.

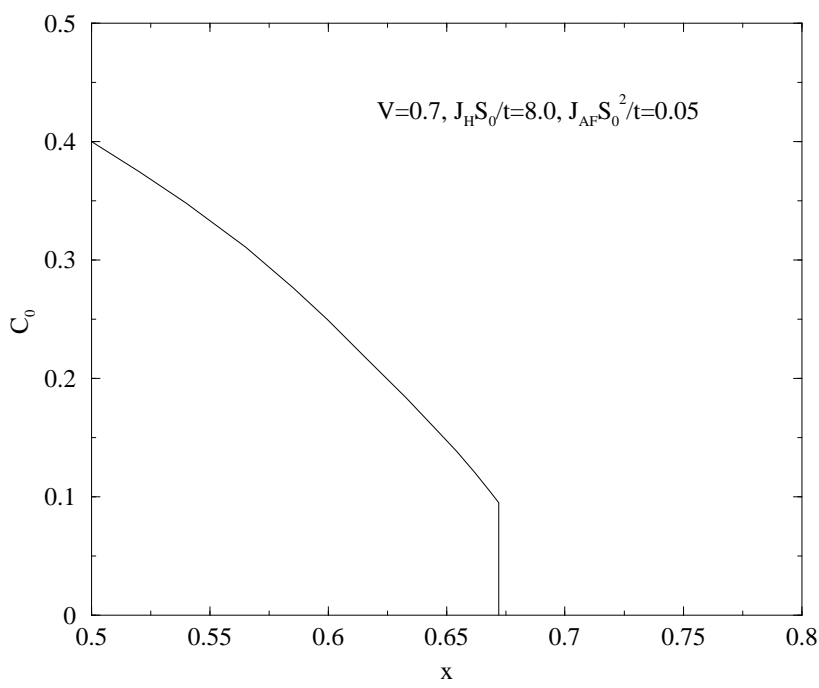

FIG. 4: Variation of charge order as $x$ is changed from 0.5 for a fixed $V=0.7$. The jump in the order parameter indicates the A-CO to A boundary

it is very close to the staggered one. This is primarily due to the absence of coherent charge transport in the c-direction making a charge uniform state in that direction nearly degenerate with a charge ordered one. We note in passing that we did not observe the well known CE phase ${ }^{24}$ in our calculation even at $x=0.5$. This is similar to the findings in the $V=0$ case stud-

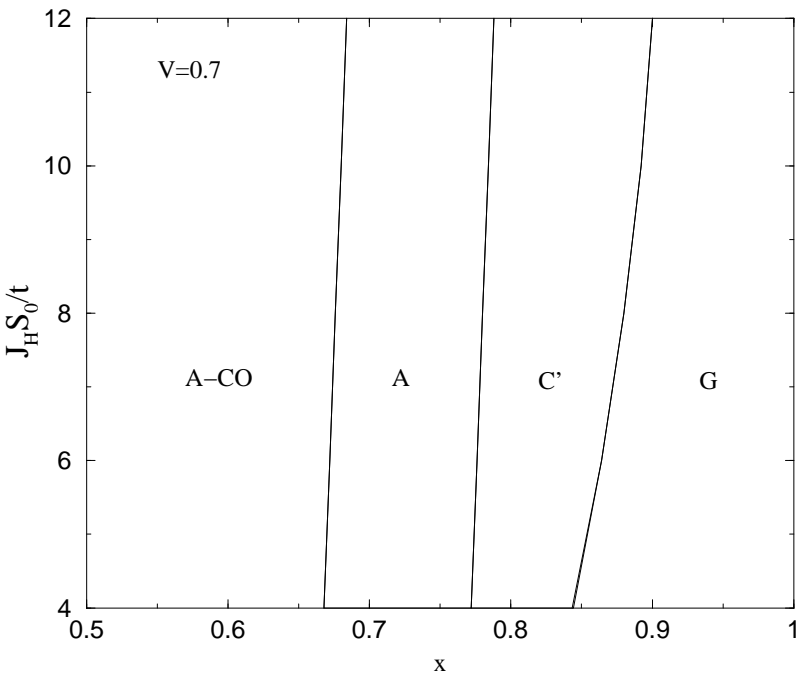

FIG. 5: Phase diagram, in the $J_{H} S_{0}$ versus filling $x$ plane. The terms A, $\mathrm{C}^{\prime}, \mathrm{G}, \mathrm{CO}$ etc. are explained in the text.

ied earlier ${ }^{7.8}$. The region of no long range order just above the A-CO coexistence reported by both Ling et al. and Coldea et al. is beyond the scope of the treatments here. It is at this point useful to note that a region of two phase coexistence is not very stable against long range Coulomb interactions and in real systems one would probably observe a microscopically phase separated mixture of one phase in another. The region of no LRO could well be such a region, around the $\mathrm{A}-\mathrm{CO}$ to $\mathrm{A}$ transition and with the competing $\mathrm{C}^{\prime}$ phase energetically very close by.

In the absence of $\mathrm{CO}$ the phases $\mathrm{A}$ and $\mathrm{C}^{\prime}$ are both orbitally ordered ${ }^{7}$ and we look for this in the presence of the near-neighbour Coulomb term also. We do indeed find very similar orbital occupancies here too. There is a predominant occupancy in the $d_{3 z^{2}-r^{2}}$ orbital over the $d_{x^{2}-y^{2}}$ orbital indicating orbital ordering. The occupancies in the two orbitals must add up to the total electron density $\left(\frac{1-x}{4}\right)$. The effect of charge ordering is seen to be small, shown in Fig. 6, reducing the orbital order (measured by the imbalance in the orbital occupancies) in the A-CO phase from its value in the absence of $\mathrm{CO}$. There is no effect in orbital occupancies at the A-CO to A boundary, they smoothly continue into the pure A-phase. The presence of orbital order here is not contingent upon the underlying lattice distortion or JT effects. This conforms to the recent observation of Wilkins et al ${ }^{12}$ that even without any JT distortion, there is a pronounced orbital order. In the region of $\mathrm{C}^{\prime}$-phase, 


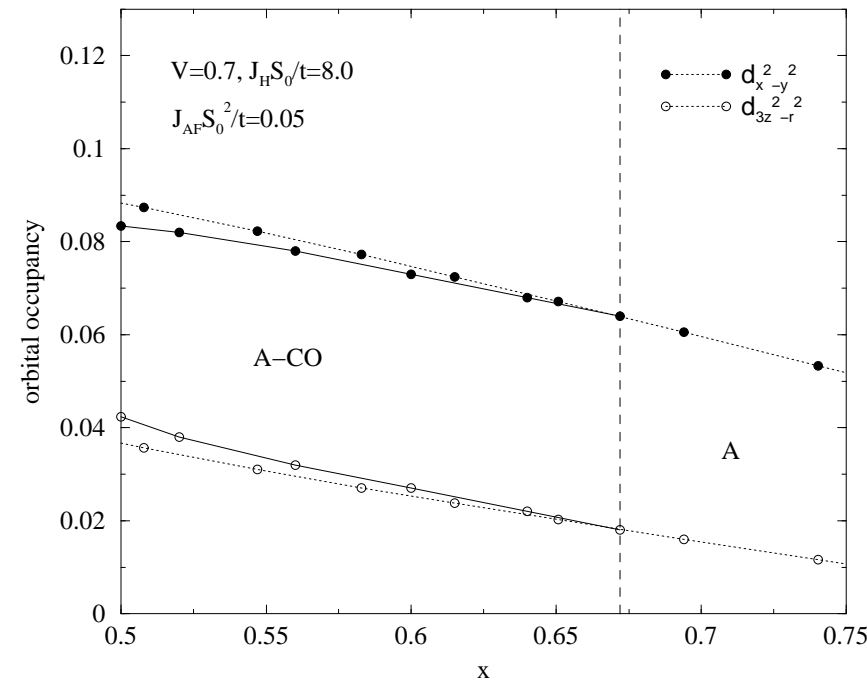

FIG. 6: Orbital occupancies in the A-CO and A-phases. The upper curves are for $d_{3 z^{2}-r^{2}}$ while the lower ones are for the $d_{x^{2}-y^{2}}$ orbitals. The dotted lines are for comparison with $V=0$ situation where no $\mathrm{CO}$ is present.

the orbital order is identical (predominantly $d_{3 y^{2}-r^{2}}$ over $d_{z^{2}-x^{2}}$ ) to the one reported earlier ${ }^{7}$ for the undistorted bilayer system and are not shown here. We have looked into the effect of an inter-orbital repul- sion term on the phase diagram and charge ordering and except for a slight enhancement of the orbital order, found it to have little effect on the phase diagram. The intra-orbital Coulomb term is known to have very little effect ${ }^{16}$ in the mean-field theory in the region of large $\mathrm{J}_{H}$ and we haven't considered it in the above.

\section{CONCLUSION}

Motivated by the observation of charge ordering in the region $x \geq 0.5$ in the bilayer systems, we have investigated it from a model that has been quite useful in understanding the overdoped manganites. The charge order has been found in our calculation in the same region where it has been seen experimentally. There is also a region of phase separation abutting the experimentally observed no LRO region. We observe orbital order even in the absence of JT distortion as reported recently in the same region of doping. It would be interesting to see more experiments on the region of no LRO in the phase diagram. The possibility of canting of spins is yet not ruled out and we have seen trends for it for very large Hund's coupling in our calculations.

Acknowledgement We acknowledge useful discussions with M. Capezzali and G. V. Pai.
1 C. D. Ling et al., Phys. Rev. B 62, 15096 (2000).

${ }^{2}$ Y. Moritomo et al., J. Phys. Soc. Jpn. 67, 405 (1998).

3 T. Kimura et al., Phys. Rev. B 58, 11081 (1998).

${ }^{4}$ K. Hirota et al., J. Phys. Soc. Jpn. 67, 3380 (1998).

5 A. Coldea et al., Phys. Rev. Lett. 89, 277601 (2002).

${ }^{6}$ X. Qiu et al., cond-mat/0307652

7 Tulika Maitra and A. Taraphder, Europhys. Lett. 65, 262 (2004).

8 R. Maezono and N. Nagaosa, Phys. Rev. B. 61, 1825 (1998); R. Maezono, S. Ishihara and N. Nagaosa, Phys. Rev. B. 58, 11583 (1998).

9 M. Kubota et al., J. Phys. Chem. Solids 60, 116 (1999); M. Kubota et al., cond-mat/9902288

10 D. N. Argyriou et al., Phys. Rev. B. 61, 15269 (2000).

11 T. Chatterjee et al. Phys. Rev. B. 61, 570 (2000).

12 S. B. Wilkins et al., cond-mat/0412435

13 T. Akimoto et. al, Phys. Rev. B 57, R5594 (1998).

14 J. van den Brink and D. Khomskii, Phys. Rev. Lett 82, 1016 (1999).

15 Tulika Maitra and A. Taraphder, Europhys. Lett. 59,
896 (2002).

16 T. Hotta, A. Malvezzi and E. Dagotto, Phys. Rev. B 62, 9432 (2000); E. Dagotto, T. Hotta and A. Moreo, Phys. Reports 344, 1 (2001).

17 H. Fujioka et al., cond-mat/9902253 K. Hirota et al., cond-mat/0104535

18 C. Zener, Phys. Rev. 82, 403 (1951); P. W. Anderson and H. Hasegawa, ibid 100, 675 (1955).

19 J. C. Slater and G. F. Koster, Phys. Rev. 94, 1498 (1954).

20 Tulika Maitra and A. Taraphder, Phys. Rev. B, 66, 174416 (2003).

21 G. Jackeli, N. B. Perkins and N. M. Plakida, Phys. Rev. B 62, 372 (2001).

22 S. Okamoto, S. Ishihara and S. Maekawa, Phys. Rev. B 63, 104401 (2001).

23 P. G. de Gennes, Phys. Rev. 118, 141 (1960).

${ }^{24}$ K. Kanamori, J. Appl. Phys. 14S (1960) ; J. B. Goodenough, Phys. Rev. 100, 564 (1955). 